\title{
Correlation Between Vasoactive-Inotropic Score and Postoperative Acute Kidney Injury after Cardiovascular Surgery
}

\author{
Kelong Hou ${ }^{1}$, Qi Chen ${ }^{1}$, Xiaojie Zhu ${ }^{1}$, Xiao Shen ${ }^{2}$, Lei Zou ${ }^{2}$, Xinwei Mu², and Xiangdong Sun ${ }^{1}$ \\ ${ }^{1}$ Department of Intensive Care Unit, The Third People's Hospital of Bengbu, Bengbu, Anhui Province, China; ${ }^{2}$ Department of \\ Intensive Care Unit, Nanjing First Hospital, Nanjing Medical University, Nanjing, Jiangsu Province, China
}

\section{ABSTRACT}

Background: Acute kidney injury (AKI) is a common complication of cardiovascular surgery. The aim of this study was to investigate the correlation between Vasoactive-Inotropic Score (VIS) and postoperative acute kidney injury in adult patients with cardiovascular surgery.

Methods: We retrospectively reviewed the data of 1935 adult patients who underwent cardiovascular surgery between September 2017 and May 2019. The data of patients included demographic data, laboratory findings, intraoperative details, and postoperative clinical outcomes. We calculated VISmax by using the highest doses of vasoactive and inotropic medications during the first $24 \mathrm{~h}$ after cardiovascular surgery. Logistic regression model was used to evaluate whether the VIS-max was independently associated with postoperative AKI. Additionally, improvements in risk reclassification and discrimination were evaluated by calculating the net reclassification improvement (NRI), C-index and the integrated discrimination improvement (IDI) with the addition of the VIS-max to a baseline model of the Society of Thoracic Surgeons (STS) score for analyzing the association of VIS-max with postoperative AKI.

Results: In 1935 patients, 291 patients (15.0\%) developed postoperative AKI from the second to seventh day after cardiovascular surgery, and 30 patients $(1.6 \%)$ needed renal replacement therapy (RRT). In 291 patients with AKI, 3 patients $(0.2 \%)$ with AKI class 1,12 patients $(0.6 \%)$ with AKI class 2, and 15 patients $(0.8 \%)$ with AKI class 3 needed RRT. Multivariate logistic regression analysis showed that VIS-max was associated with postoperative AKI (odds ratio [OR]: 1.19, $95 \%$ confidence interval $[\mathrm{CI}]: 1.11-1.34, P<0.001)$ and the need for RRT in AKI patients (OR: 1.29, 95\%CI: 1.01-1.83, $P=0.007$ ). The area under the ROC curves (AUROC) of VIS-max combining STS score for predicting postoperative AKI (AUROC: $0.84,95 \% \mathrm{CI}: 0.81-0.87, P<0.001$ ) and need of RRT (AUROC: 0.91, 95\% CI: 0.86-0.96, $P<0.001$ ) significantly were higher than the AUC of VIS-max, STS score and

Received December 15, 2020; received in revised form fanuary 30, 2021; accepted February 2, 2021

Correspondence: Xiangdong Sun and Xiaojie Zhu, Department of Intensive Care Unit, The Third People's Hospital of Bengbu, Bengbu 233000, Anbui Province, China (e-mail: sxd640610@sina.com,757773536@qq.com)
EuroSCORE. Inclusion of VIS-max into basic risk model of STS score provided an increase in all indexes of prognostic accuracy for postoperative AKI and need of RRT: C-statistic: 0.721, NRI: $21.8 \%$, IDI: 4.9\%; and C-statistic: 0.745, NRI: $24.7 \%$, IDI: $5.6 \%$, respectively.

Conclusion: VIS-max is an independent predictor of postoperative AKI in adult patients after cardiovascular surgery and increases prognostic accuracy of STS score, allowing a risk reclassification.

\section{INTRODUCTION}

Acute kidney injury (AKI) is a common and serious complication in patients undergoing both cardiac surgery and vascular surgery, occurring in $20 \%$ to $70 \%$ of cases, depending on the type of surgery and the definition of AKI used [Nadim 2018]. Cardiac and vascular surgery-associated AKI (CVSAKI) is associated with poor outcomes, including a prolonged hospital stay, an increased duration of mechanical ventilation, and high mortality [Yuan 2019]. It significantly increases mortality and morbidity, and to decrease these adverse events, many studies have focused on identifying preventative strategies as well as risk scores or biomarkers for AKI after cardiac and vascular surgery. Some heart surgery risk scores are used in cardiac surgery, such as the Society of Thoracic Surgeons (STS) mortality risk score, Cleveland Clinic Score, and the CRATE score [Jorge-Monjas 2016; Ranucci 2018; Che 2019; Badhwar 2018]. However, it is not clear whether these scoring systems have good discrimination in estimating the risk of CVS-AKI and the changes of renal function in patients undergoing both cardiac surgery and vascular surgery.

In 1995, the inotrope score (IS) was initially described in a study by Wernovsky and colleagues [Wernovsky 1995]. This score was to quantify amount of cardiovascular support and used in clinical research as a measure of illness severity in patients undergoing cardiac surgery [Gruenwald 2008]. In 2010, Gaies et al. [Gaies 2010] proposed expanding the inotropic score to include other commonly vasoactive medications including milrinone, vasopressin and norepinephrine, calling it a vasoactive-inotrope score (VIS). And then some studies showed that VIS had good predictive accuracy and discrimination capability for poor short-term outcomes in infant, pediatric, or adolescent patients after cardiac surgery [Butts 2012; Gaies 2014; Kim 2015; Garcia 2016]. More recently, some research reported that VIS also showed similar 
predictive accuracy in an adult population that underwent cardiac surgery [Yamazaki 2018; Koponen 2019]. The maximum VIS in the first 24 postoperative hours (VIS-max) was proved to be a good predictor for poor outcomes after cardiac surgery [Koponen 2019]. However, there are only limited data to support the use as a predictor for postoperative AKI in the early postoperative period.

Therefore, we undertook this retrospective study with the aim of determining whether the addition of VIS-max to STS score could be better to stratify the risk of CVS-AKI in a population that underwent cardiac surgery and vascular surgery.

\section{MATERIALS AND METHODS}

Setting and study population: The study was approved by the institutional review board of Nanjing First Hospital, where the requirement to obtain patient consent was waived by the ethics committee because the data analysis was retrospective.

We performed a single-center retrospective cohort study screening all cardiac and vascular surgery patients admitted to the surgical ICU at Nanjing First Hospital between September 2017 and May 2019. The screened patients underwent cardiovascular surgeries, covering coronary artery bypass grafting (CABG) surgery, valve replacement or repair, heart transplantation, ascending aorta or aortic arch operations, and other cardiac surgery. They were included in this study. Patients were excluded if they met the following criteria: age $<18$ years old, chronic kidney diseases (eGFR $<30 \mathrm{~mL} / 1.73 \mathrm{~m} 2$ ), preoperative AKI (the definition and stage of AKI were based on the Kidney Disease: Improving Global Outcomes guidelines [KDIGO] in 2012) [Kellum 2013], death within postoperative 24 hours, nephrectomy, or renal artery stenosis, and cases with missing data. In addition, patients who developed postoperative AKI within 24 hours also were excluded because the VISmax score was calculated by the maximum dose of vasoactive and inotropic medications on POD-1, and this would cause VIS-max on POD-1 to predict AKI on POD-1 early.

Perioperative clinical variables: Demographic and clinical data were collected from medical records. These data included preoperative data, operative details and postoperative results, including age, gender, body mass index (BMI), preexisting clinical conditions, STS score, the European heart surgery risk assessment system (EuroSCORE), estimated glomerular filtration rate (eGFR), serum creatinine (Scr), hemoglobin $(\mathrm{Hb})$, albumin, serum uric acid, serum neutrophil gelatinaseassociated lipocalin (NGAL), surgery type, operation time, aortic cross-clamp time, cardiopulmonary bypass $(\mathrm{CPB})$ time, red blood cell (RBC) units, acute physiology and chronic health evaluation II score (APACHE II score), sequential organ failure assessment (SOFA) score, VIS-max, lactate, mean arterial pressure (MAP), central venous pressure (CVP), and fluid balance.

We recorded the vasoactive and inotropic medications used to maintain hemodynamic stability after cardiovascular surgery in all the patients and calculated the VIS-max using the maximum dosing rates of vasoactive and inotropic medications during the first 24 postoperative hours at ICU admission. (VIS = dopamine dose $[\mu \mathrm{g} / \mathrm{kg} / \mathrm{min}]+$ dobutamine $[\mu \mathrm{g} /$ $\mathrm{kg} / \mathrm{min}]+100 \times$ epinephrine dose $[\mathrm{\mu g} / \mathrm{kg} / \mathrm{min}]+50 \times$ levosimendan dose $[\mu \mathrm{g} / \mathrm{kg} / \mathrm{min}]+10 \times$ milrinone dose $[\mu \mathrm{g} / \mathrm{kg} / \mathrm{min}]$ $+10000 \times$ vasopressin [units $/ \mathrm{kg} / \mathrm{min}$ ] + 100×norepinephrine dose $[\mu \mathrm{g} / \mathrm{kg} / \mathrm{min}$ ] [Gaies 2010; Koponen 2019].

Outcome variables: The primary outcome was the development of AKI after cardiovascular surgery. The authors defined AKI, according to the KDIGO criteria [Kellum 2013]. AKI was diagnosed as any of the following: increased in Scr by $\geq 0.3 \mathrm{mg} / \mathrm{dl}(\geq 26.5 \mu \mathrm{mol} / \mathrm{l})$ within 48 hours; or increased in Scr to $\geq 1.5$ times baseline, which was known or presumed to have occurred within the prior 7 days; or urine volume $<0.5 \mathrm{ml} /$ $\mathrm{kg} /$ hour for 6 hours. Postoperative clinical outcome variables included the need for renal replacement therapy (RRT)(RRT was initiated if one of the following criteria were met: blood urea nitrogen $\geq 100 \mathrm{mg} / \mathrm{dL}$, serum potassium $>6 \mathrm{mmol} / \mathrm{L}$, serum bicarbonate $<12 \mathrm{mmol} / \mathrm{L}$ or $\mathrm{pH}<7.15, \mathrm{PaO} 2 / \mathrm{FiO} 2$ ratio $<200$, or chest radiograph compatible with pulmonary edema) [Lumlertgul 2018], mechanical ventilation time, the length of ICU stay, length of hospital stay, ICU mortality, hospital mortality, 30-days and 90-days all-cause mortality.

Statistical analysis: Two standard statistical packages (SPSS software version 19.0, IBM, NY, USA) and STATA software version 11.0, StataCorp, TX, USA) were used to analyze the data. In all cases, a $P$-value of $<0.05$ was considered statistically significant. The sample size was calculated with $\mathrm{G}^{*}$ Power 3.1 (http://www.gpower.hhu.de/). A sample size of 503 or more patients was required with a power of 0.95 and type I error of 0.05 . This was calculated under the assumption that the expected odds ratio (OR) for postoperative AKI in patients, who underwent cardiovascular surgeries with increased VISmax would be 1.5 , and the incidence of postoperative AKI in patients with increased VIS-max would be $20 \%$.

Missing data were present in $<4 \%$, including preoperative NGAL $(N=42)$ and preoperative Scr $(N=19)$, and these cases were removed. There was no missing data regarding VIS-max and RRT. The normality of the distribution of continuous variables was tested using the Kolmogorov-Smirnov test. Normally distributed data are expressed as the means \pm standard deviation and were compared by unpaired t tests. Non-normally distributed data are expressed as the medians (interquartile ranges) and were compared by the MannWhitney U test or Kruskal-Wallis test. Categorical variables are presented as absolute numbers or percentages and were analyzed using the $\chi^{2}$ test or Fisher's exact test.

Logistic regression model was used to identify preoperative, intraoperative, and postoperative factors for AKI and evaluate whether the VIS-max was independently associated with postoperative AKI. Univariable logistic regression analysis was used first to identify possible risk factors for AKI. The 27 predictor variables that were significant on univariable analysis were selected for inclusion into the multivariable analysis. (Table 3 ) Independent predictor variables were selected by performing a backward stepwise variable elimination process with a significant criterion of $P<0.05$. In addition, similarly, as a subgroup analysis, the univariable and multivariable logistic analysis was performed in the patients developing postoperative AKI with RRT and without RRT. Data were presented as OR and $95 \%$ confidence intervals 
Table 1. Characteristics of patients according to postoperative acute kidney injury

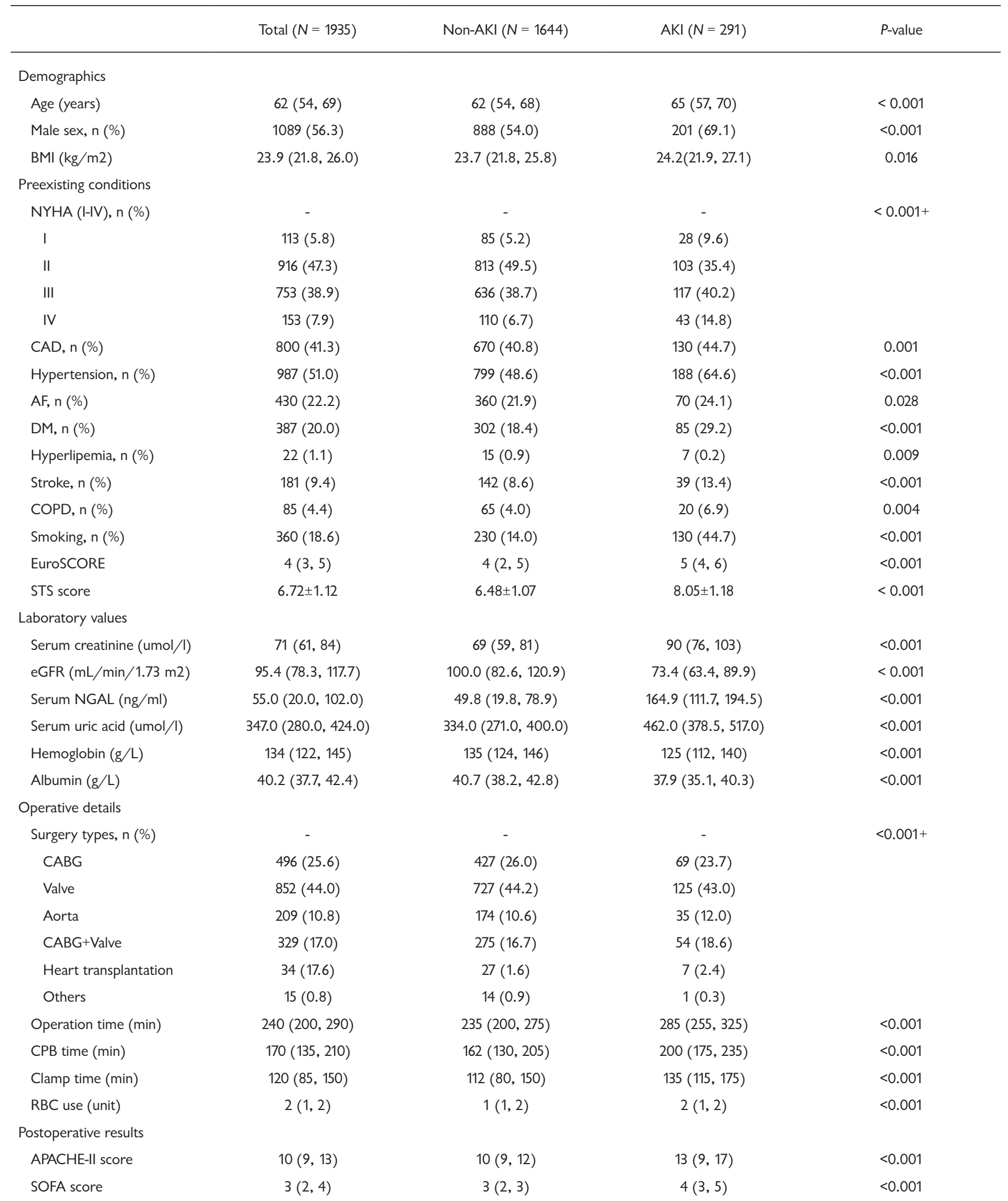




$\begin{array}{lcccr}\text { VIS-max within 24h } & 7(5,9) & 5(4,8) & 10(8,13) & <0.001 \\ \text { MAP within 24h (mmHg) } & 71.0(68.0,74.6) & 72.0(69.0,75.0) & 68.6(64.3,73.0) & <0.001 \\ \text { CVP within 24h (mmHg) } & 8.0(6.0,10.0) & 8.0(6.0,9.0) & 11.0(10.0,12.0) & <0.001 \\ \text { Lactate within 24h (mmol/l) } & 1.6(1.2-2.1) & 1.6(1.2,2.1) & 2.0(1.0,3.1) & 0.013 \\ \text { Fluid balance within 24h (ml) } & -231(-630,156) & -274(-651,105) & 146(-475,458) & <0.001\end{array}$

BMI, body mass index; NYHA, New York Heart Association; CAD, coronary artery disease; AF, atrial fibrillation; DM, diabetes mellitus; COPD, chronic obstructive pulmonary disease; EuroSCORE, the European heart surgery risk assessment system; STS score, the Society of Thoracic Surgeons Score; CABG, coronary artery bypass grafting; NGAL, neutrophil gelatinase-associated lipocalin; CPB, cardiopulmonary bypass; RBC, red blood cell; APACHE II, Acute Physiology and Chronic Health Evaluation Score II; SOFA, sequential organ failure assessment. VIS-max, maximum vasoactive inotropic score in the first postoperative $24 \mathrm{~h}$; MAP, mean arterial pressure; CVP, central venous pressure.

Table 2. Clinical outcomes in patients with postoperative AKI

\begin{tabular}{|c|c|c|c|c|}
\hline RRT, n (\%) & $30(1.6)$ & $0(0)$ & $30(10.3)$ & $<0.001++$ \\
\hline ICU stays (days) & $1(1,2)$ & $1(1,2)$ & $4(2,7)$ & $<0.001$ \\
\hline Hospital stay (days) & $16(13,20)$ & $16(13,19)$ & $21(15,26)$ & $<0.001$ \\
\hline Hospital mortality, n (\%) & $12(0.6)$ & $7(0.4)$ & $5(0.3)$ & 0.029 \\
\hline ICU mortality, n (\%) & $9(0.5)$ & $5(0.3)$ & $4(1.4)$ & 0.045 \\
\hline 30-days All-cause mortality, n (\%) & $20(1.0)$ & $13(0.8)$ & $7(2.4)$ & 0.012 \\
\hline 90-days All-cause mortality, n (\%) & $31(1.6)$ & $20(1.2)$ & $11(3.8)$ & 0.001 \\
\hline
\end{tabular}

RRT, renal replacement therapy; MV, mechanical ventilation; ICU, intensive care unit. ++ Fisher exact test.

(CI). The calibration of the final multivariable logistic regression model was evaluated using Hosmer-Lemeshow goodness-of-fit test. Good calibration was indicated by a low $\chi^{2}$ and a high $P$ value $>0.05$.

The discriminatory power was quantified by the area under the receiver operating characteristic curve (AUROC). To compare the predictive ability of VIS-max + STS score, VIS-max, STS score, and EuroSCORE, AUCs of the variables were compared using the nonparametric DeLong's methods [DeLong 1988]. (Figure 2) Additionally, the net reclassification improvement (NRI), C-statistic and integrated discrimination improvement (IDI) were evaluated to determine whether the VIS-max significantly improved risk reclassification and discrimination for CVS-AKI and need of RRT when added to a baseline model (the STS score).

\section{RESULTS}

During the study period, 2173 consecutive cardiovascular surgery patients were evaluated, and 238 patients were excluded (105 for postoperative AKI within the first 24 hours, 61 for missing data, 51 for CKD, 6 for preoperative AKI, 6 for nephrectomy, 5 for age $<18,3$ for death within postoperative
$24 \mathrm{~h}$ and 1 for renal artery stenosis). Thus, 1935 patients were included in this study, as shown in Figure 1. (Figure 1) In all patients, 1644 patients (85.0\%) presented with no AKI and 291 patients $(15.0 \%)$ developed postoperative AKI as defined by the KDIGO criteria with stage $1(N=196,10.1 \%)$, stage $2(N$ $=58,3.0 \%)$, or stage $3(N=37,1.9 \%)$ from POD-2 to POD-7, and 30 patients (1.6\%) required RRT. (Table 6) Demographic, preoperative, intraoperative and postoperative variables and clinical outcomes were described in Tables 1 and 2 .

Patients with postoperative AKI had higher STS score and EuroSCORE and were older, more often male, more likely to have preexisting $\mathrm{CAD}$, hypertension, $\mathrm{AF}, \mathrm{DM}$, hyperlipemia, stroke, COPD, and smoking. These patients with AKI had higher serum creatinine, NGAL, and uric acid level; they also had lower preoperative $\mathrm{Hb}$, albumin and eGFR before cardiovascular surgery. Longer operation time, CPB time, aortic cross-clamp time and more RBC units' infusion also were associated with postoperative AKI. During the first 24 hours after cardiovascular surgery, patients developing AKI had more serious clinical condition, and needed more vasoactive and inotropic medication supports (VIS-max: 10 [Wernovsky 1995; Kim 2015] vs. 5 [Ranucci 2018; Wernovsky 1995], $\mathrm{P}<$ 0.001). These patients had higher APACHE II score, SOFA score, lactate level, CVP and fluid balance, and lower MAP. 
Table 3. Univariable and multivariable logistic regression analysis of risk factors for postoperative AKI

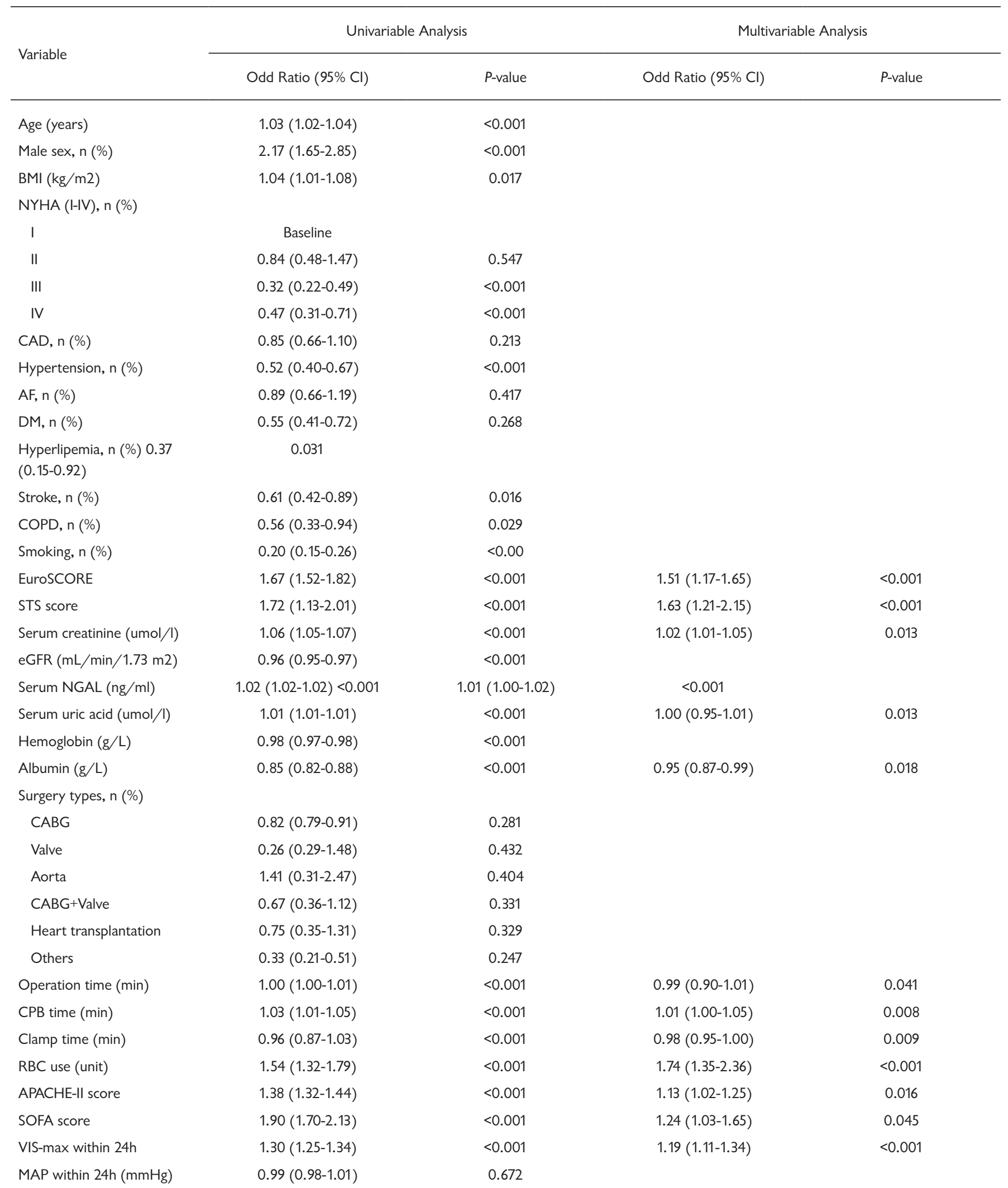




\begin{tabular}{lllll} 
CVP within 24h $(\mathrm{mmHg})$ & $1.77(1.66-1.90)$ & $<0.001$ & $1.53(1.41-1.78)$ & $<0.001$ \\
Lactate within 24h $(\mathrm{mmol} / \mathrm{l})$ & $1.48(1.32-1.66)$ & $<0.001$ & $1.31(1.02-1.66)$ & 0.037 \\
Fluid balance within $24 \mathrm{~h}(\mathrm{ml})$ & $1.00(1.00-1.03)$ & $<0.001$ & $1.02(1.00-1.02)$ & 0.025 \\
\hline
\end{tabular}

BMI, body mass index; NYHA, New York Heart Association; CAD, coronary artery disease; AF, atrial fibrillation; DM, diabetes mellitus; COPD, chronic obstructive pulmonary disease; EuroSCORE, the European heart surgery risk assessment system; STS score, the Society of Thoracic Surgeons Score; CABG, coronary artery bypass grafting; NGAL, neutrophil gelatinase-associated lipocalin; CPB, cardiopulmonary bypass; RBC, red blood cell; APACHE II, Acute Physiology and Chronic Health Evaluation Score II; SOFA, sequential organ failure assessment. VIS-max, maximum vasoactive inotropic score in the first postoperative $24 \mathrm{~h}$; MAP, mean arterial pressure ; CVP, central venous pressure

(Table 1) In clinical outcomes, patients with postoperative AKI had longer ICU stay, hospital stay, mechanical ventilation time, and higher hospital mortality, ICU mortality, 30-days all-cause mortality and 90-days all-cause mortality when compared with those without AKI. (Table 2)

All variables were analyzed in the univariate logistic regression model to determine the statistically significant factors that were contributed to AKI, and these independent risk factors were further taken into the multivariate logistic regression. As shown in Table 3, 16 variables were established by multivariate logistic regression as independent risk factors for the development of postoperative AKI. A higher VIS-max within the first 24 hours after cardiovascular surgery (odds ratio [OR] 1.19, $95 \%$ confidence interval $[\mathrm{CI}], 1.11$ to $1.34, P<0.001)$ and STS score (odds ratio [OR] 1.63, 95\% confidence interval [CI], 1.21 to $2.15, P<0.001)$ were associated with AKI. Calibration with the Hosmer-Lemeshow test showed a good fit $\left(\chi^{2}=9.13, P=\right.$ 0.331 , and $\chi^{2}=8.14, P=0.275$, respectively). In addition, stepwise forward variable selection method also showed that VIS$\max (\mathrm{OR} 1.29,95 \% \mathrm{CI}, 1.01$ to $1.83, P=0.007)$ and STS score (OR $1.36,95 \% \mathrm{CI}, 1.14$ to $2.35, P=0.017$ ) were associated with the need for RRT in patients with postoperative AKI (Table 4), and Hosmer-Lemeshow test also showed a good fit $\left(\chi^{2}=5.74, P\right.$ $=0.677$, and $\chi^{2}=7.81, P=0.536$, respectively). (Table 4)

The discrimination power of VIS-max+STS score for postoperative AKI underwent cardiovascular surgery was better than VIS-max, STS score, and EuroSCORE (AUC of VISmax+STS score vs. VIS-max: 0.84 vs $0.81, P<0.001$, AUC of VIS-max+STS score vs STS score: 0.84 vs. $0.74, P<0.001$, AUC of VIS-max+STS score vs. EuroSCORE: 0.84 vs. $0.71, P$ $<0.001$ by Delong method) (Figure 2). Furthermore, the AUC of VIS-max+STS score was significantly higher than VIS-max, STS score, and EuroSCORE (AUC of VIS-max+STS score vs. VIS-max: 0.91 vs. $0.88, P<0.001$, AUC of VIS-max+STS score vs. STS score: 0.91 vs. $0.84, P<0.001$, AUC of VISmax+STS score vs. EuroSCORE: 0.91 vs. $0.73, P<0.001$ by Delong method). (Figure 3) In addition, as shown in Table 6 , the inclusion of VIS-max to the risk model of STS score produced a significant increase in NRI and IDI for both AKI and need of RRT (C-statistic: 0.721 , NRI: $21.8 \%$, IDI: $4.9 \%$; and C-statistic: 0.745 , NRI: $24.7 \%$, IDI: 5.6\%, respectively).

\section{DISCUSSION}

To our knowledge, this is the first study validating the association between VIS and postoperative AKI in adult

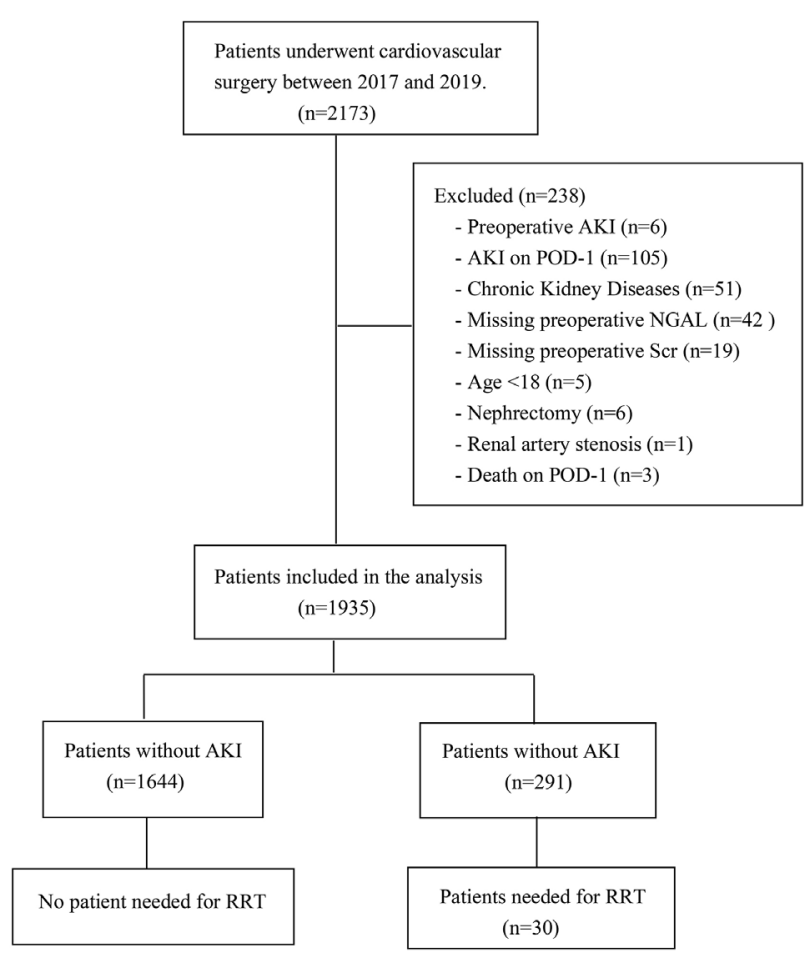

Figure 1. Flowchart diagram of inclusion

patients who had undergone cardiovascular surgery. In this retrospective study, we reported a new score called VIS-max and added to a baseline model (the STS score) for predicting postoperative AKI and need of RRT after cardiovascular surgery. A significant independent association was found between VIS-max+STS score and AKI from the second day to seventh day after surgery, and the predictive power of VISmax+STS score for postoperative AKI and need of RRT were better than VIS-max, STS score, and EuroSCORE. Furthermore, the inclusion of VIS-max to the risk model of STS score produced a significant increase in NRI and IDI for both AKI and need of RRT.

The possible explanation of the association between VISmax and postoperative AKI may be divided into two aspects. On one hand, indirectly, the association of VIS-max and AKI may be illustrated by the hemodynamic disturbance. The cause of AKI after cardiovascular surgery is multifactorial and incompletely understood [Nadim 2018]. Possible causative factors include hemodynamic perturbations, ischemia-reperfusion 
Table 4. Univariable and multivariable logistic regression analysis of risk factors for need for RRT in patients with postoperative AKI

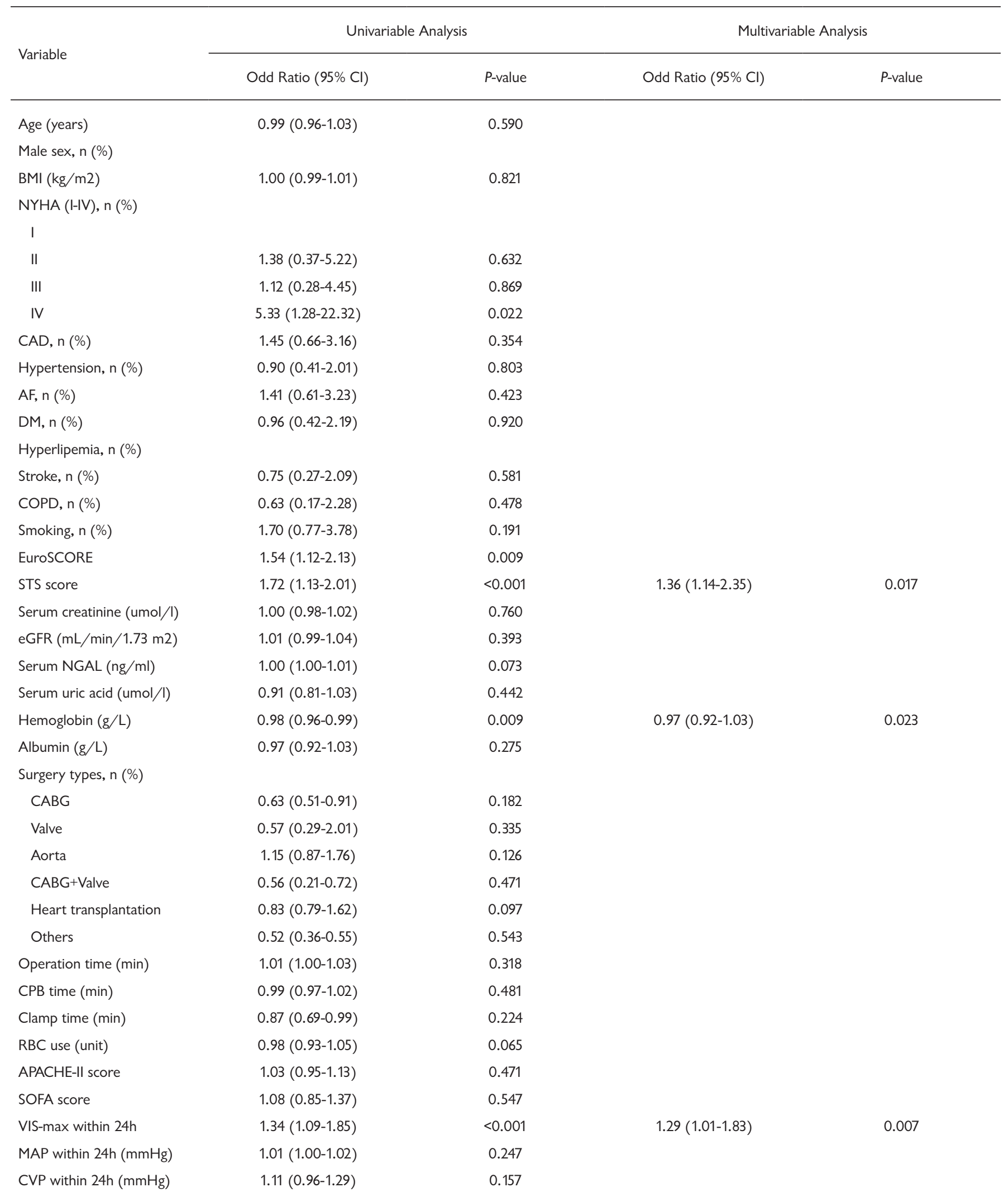




$\begin{array}{lllll}\text { Lactate within } 24 \mathrm{~h}(\mathrm{mmol} / \mathrm{l}) & 1.16(1.03-1.30) & 0.013 & 1.03(0.99-1.18) & 0.037 \\ \text { Fluid balance within } 24 \mathrm{~h}(\mathrm{ml}) & 1.00(1.00-1.00) & 0.730 & \end{array}$

BMI, body mass index; NYHA, New York Heart Association; CAD, coronary artery disease; AF, atrial fibrillation; DM, diabetes mellitus; COPD, chronic obstructive pulmonary disease; EuroSCORE, the European heart surgery risk assessment system; CABG, coronary artery bypass grafting; NGAL, neutrophil gelatinase-associated lipocalin; CPB, cardiopulmonary bypass; RBC, red blood cell; APACHE II, Acute Physiology and Chronic Health Evaluation Score II; SOFA, sequential organ failure assessment; VIS-max, maximum vasoactive inotropic score in the first postoperative $24 \mathrm{~h}$; MAP, mean arterial pressure; CVP, central venous pressure

Table 5. Risk reclassification for postoperative AKI after adding to VIS-max to the STS score

\begin{tabular}{|c|c|c|c|c|c|c|c|}
\hline Variables & Model & \multicolumn{3}{|c|}{ NRI } & \multicolumn{3}{|c|}{ IDI } \\
\hline AKI & Basic model + VIS-max & $21.8 \%$ & $17.4-25.7 \%$ & 0.024 & $4.9 \%$ & $3.9-5.7 \%$ & 0.018 \\
\hline Need of RRT & Basic model + VIS-max & $24.7 \%$ & $22.4-27.9 \%$ & 0.015 & $5.6 \%$ & $5.1-6.8 \%$ & 0.007 \\
\hline
\end{tabular}

NRI, net reclassification improvement; ref, reference; IDI, integrated discrimination improvement; STS score, the Society of Thoracic Surgeons Score

Table 6. Rates of RRT in patients with postoperative AKI class 1-3

\begin{tabular}{|c|c|c|c|c|c|c|c|c|}
\hline & AKI-1 & RRT & AKI-2 & RRT & AKI-3 & RRT & AKI 1-3 & RRT \\
\hline POD-2 & $120(6.2 \%)$ & $2(0.1 \%)$ & $38(2.0 \%)$ & $8(0.4 \%)$ & $18(0.9 \%)$ & $9(0.5 \%)$ & $176(9.1 \%)$ & $19(1.0 \%)$ \\
\hline POD-3 & $55(2.8 \%)$ & $1(0.1 \%)$ & $12(0.6 \%)$ & $3(0.2 \%)$ & $13(0.7 \%)$ & $4(0.2 \%)$ & $80(4.1 \%)$ & $8(0.4 \%)$ \\
\hline POD-6 & $1(0.1 \%)$ & $0(0)$ & $0(0)$ & $0(0)$ & $0(0)$ & $0(0)$ & $1(0.1 \%)$ & $0(0)$ \\
\hline POD-7 & $0(0)$ & $0(0)$ & $0(0)$ & $0(0)$ & $0(0)$ & $0(0)$ & $0(0)$ & $0(0)$ \\
\hline Total & $196(10.1 \%)$ & $3(0.2 \%)$ & $58(2.9 \%)$ & $12(0.6 \%)$ & 37 (1.9\%) & $15(0.8 \%)$ & 291 (15.0\%) & $30(1.6 \%)$ \\
\hline
\end{tabular}

POD, postoperative days; RRT, renal replacement therapy

injury, the activation of inflammatory pathways, and the liberation of free hemoglobin and iron [Nadim 2018; Vanmassenhove 2017]. Among these factors, hemodynamic perturbations, especially renal hypoperfusion, were considered to be an important determinant in the development of AKI after cardiovascular surgery [Nadim 2018; Vanmassenhove 2017]. In patients with AKI after cardiovascular surgery, vasoactive and inotropic medications often are used to treat hypotension and cardiovascular dysfunction during the perioperative period. In other words, the VIS is to quantify the amount of cardiovascular support, and a higher VIS score may indicate more severe hemodynamic disorder [Gaies 2010; Gaies 2014]. Thus, patients who had a higher VIS score were more likely to developing postoperative AKI because of hemodynamic disturbance, and the VIS could be regarded as one predicting marker. On the other hand, directly, the high doses of vasoactive and inotropic medications, particularly catecholamines, had been shown to have a harmful effect on organ function and also cause immune-mediated injury [Bangalore 2017; Stamm 2002]. One study reported that patients, who had higher postoperative catecholamines level after cardiac surgery, had a greater frequency of AKI (31\% vs. $19.5 \%$ vs. $13.7 \%, P=0.038)$, and their AKI was more severe as defined by a need for renal replacement therapy ( $7.8 \%$ vs. $2.4 \%$ vs. $0 \%, P=0.026$ ) [Haase-Fielitz 2009]. In this view, higher VIS score indicates more need for vasoactive and inotropic drug supports and can evaluate the impairment of kidney function [Bangalore 2017]. 


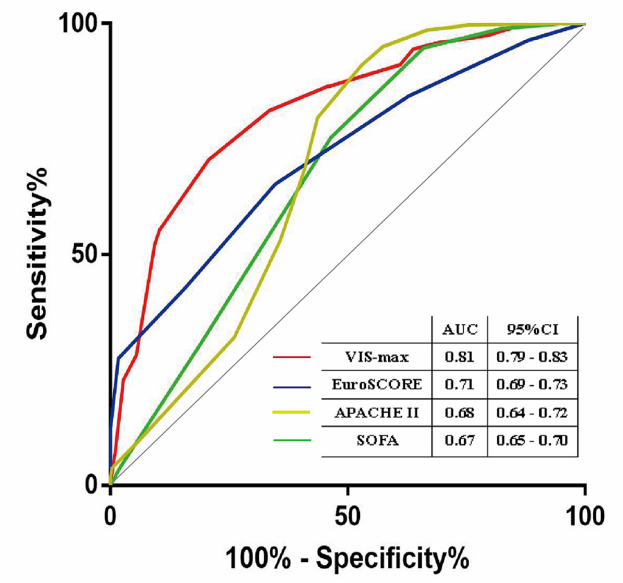

Figure 2. Comparison of the ability to predict postoperative acute kidney injury among VIS-max+STS score, VIS-max, STS sore, EuroSCORE.

In 2010, Gaies et al. [Gaies 2010] proposed a concept of vasoactive-inotrope score (VIS) based on inotrope score (IS) [Wernovsky 1995] and reported that the maximum VIS over the first 48 hours in young infants after congenital heart surgery with $\mathrm{CPB}$ was a good predictor of poor clinical outcome, including renal replacement therapy (odds ratio 8.1, 95\% CI: 3.4-19.2, $P<0.001$ ) [Gaies 2010]. After four years from then, Gaies et al. [Gaies 2014] conducted another prospective and multi-institutional cohort study that included 391 infants undergoing heart surgery with CPB. The result from multivariable logistic regression showed that maximum VIS score in the first 24 hours was association with poor outcome including postoperative AKI and renal replacement therapy (odds ratio 6.5, 95\% CI: 2.9-14.6, $P=0.001$ ). The optimal cutpoint of VIS was 20 points from the ROC curve, and the AUC was 0.79 (95\% CI: 0.73-0.85). In another retrospective study [Garcia 2016] in adolescents aged 10-18 years who underwent cardiac surgery for congenital heart disease, a maximum VIS score above 4.75 points, during the second 24 postoperative hours, had moderate sensitivity and $91 \%$ negative predictive value for short-term adverse outcome, including acute kidney injury, requiring RRT, mechanical support and death. Butts et al. [Butts 2012] also reported that maximum VIS values above 15 points, during the first 36 postoperative hours in 76 neonates, had modest correlation with long duration of mechanical ventilation, length of stay in ICU, and greater total hospital cost. In that study, although clinical outcome regarding renal function was not included, such as the incidence of postoperative AKI or need for RRT, it could still explain that VIS was associated with the clinical poor outcome and superior to low cardiac output syndrome.

In addition to focusing on postoperative VIS score, Kim et al. [Kim 2015] showed that preoperative VIS was an independent risk factor for early postoperative poor outcomes in 126 infants, who underwent arterial switch operation. The cut-off value of VIS score was 12.5 points (sensitivity $83.3 \%$, specificity $85.3 \%$ ), and the AUC was 0.85 (95\% CI: $0.64-$ 1.06). In that study, Kim et al. also found some evidence that

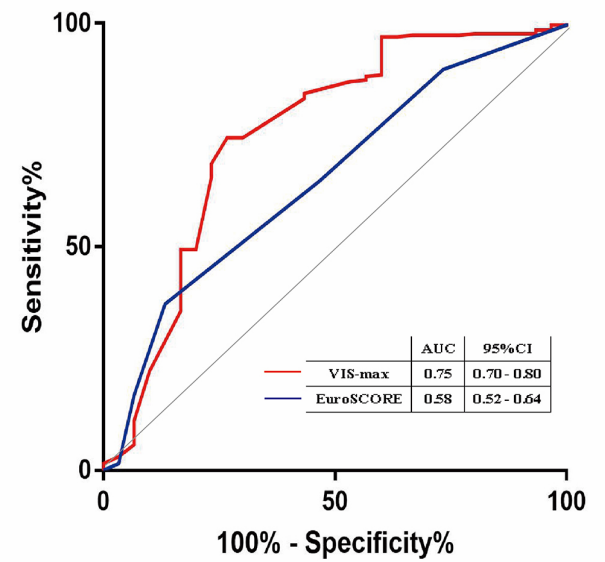

Figure 3. Comparison of the ability to predict need for RRT in patients with postoperative AKI among VIS-max+STS score, VIS-max, STS sore, EuroSCORE.

the preoperative VIS was associated preoperative ventricular function and a patient's critical condition. The patients with a higher preoperative VIS score needed more cardiovascular medication support to augment cardiac output and maintain adequate blood pressure. The preoperative hemodynamic condition was more important for the early outcomes of cardiovascular surgery [Kim 2015]. From this view, in order to clarify the predictive value of VIS for postoperative AKI, it is necessary to measure VIS before surgery or some other time, such as during operation, or within postoperative 24,48 , or 72 hours.

Most recently, Yamazaki et al. [Yamazaki 2018] focused on the VIS at the end of surgery, and the target population was changed to adult patients with cardiac surgery. After adjusting for the EuroSCORE, preoperative ejection fraction and bypass time, the result showed that a high VIS at the end of surgery was associated with a poor outcome with an adjusted odds ratio of 4.87 (95\% CI: $1.51-18.94, P=0.007)$, and a poor outcome was a composite outcome including renal dysfunction (defined by the RIFLE classification [Englberger 2011]), death, cardiac arrest, the need for mechanical ventilation support or central nervous system injury. However, in this study, the optimal cut-off value of VIS was low relatively $(5.5$ points), with sensitivity of $83 \%$ and specificity of $54 \%$, based on the ROC curve.

In our study, the inclusion of VIS-max to the STS score had better discrimination performance for postoperative AKI and need of RRT. This result was consistent with other studies. The STS score predicts the risk of operative mortality and morbidity in adults after cardiac surgery on the basis of patient demographics and clinical variables [Chang 2006]. The STS database has been used in models with endpoints other than mortality or morbidity, including renal failure. Under conditions of elective CABG, the STS score is accurate in predicting AKI [Chang 2006]. In addition, one large retrospective cohort study [Koponen 2019], including 3213 adult cardiac surgery patients, showed that maximum VIS was calculated 
using the highest doses of vasoactive and inotropic medications administered during the first 24 postoperative hours (VIS-max), and the VIS-max were divided into five categories using a $\chi^{2}$ automatic interaction detection decision tree: $0-5$, $5-15,15-30,30-45$ and $>45$ points. That study showed that VIS-max had better prediction accuracy for the composite outcome including kidney injury than SOFA, APACHE II and SAPS II score (AUC of VIS-max: 0.72, 95\% CI: 0.69-0.75, $P$ $<0.01$ ), and patients whose VIS-max score $>45$ points needed for renal dialysis therapy more likely [Koponen 2019].

In addition to being able to predict postoperative AKI, VISmax+STS score also can act as one tool to guide treatment in patients with AKI. For some conditions, such as renal failure, pulmonary oedema or severe hyperkalaemia not responsive to conservative therapy, RRT can be lifesaving [Vanmassenhove 2017]. However, the start time of RRT is a matter of ongoing debate, and it is important to use time factors, biochemical factors, or clinical characteristics to define early versus late start of RRT [Vanmassenhove 2017]. In our study, a higher VIS-max was associated with need of RRT in patients with postoperative AKI (OR: $1.29,95 \%$ CI: 1.01-1.83, $P=0.007)$, especially if VIS-max were greater than 12.5 points (AUC: $0.80,95 \% \mathrm{CI}$ : $0.80-0.95, P<0.001)$. Additionally, the inclusion of VIS-max to the risk model of STS score produced a significant increase for predicting AKI and need of RRT. This result was consistent with other studies [Gaies 2010; Gaies 2014; Garcia 2016]. In consequence, VIS-max may become one useful marker for determining the start time of RRT in combination with clinical condition, and that will improve recovery of renal function in patients with postoperative AKI. Further work is necessary to understand more precisely how VIS-max functions as a predictor of postoperative AKI at different points in the perioperative period, and how to determine the timing of RRT, according to the optimal value of VIS-max and clinical condition of patients, especially in the early stages of AKI.

In our study, patients with postoperative AKI had lower MAP $(68.6 \mathrm{mmHg}$ vs. $72.0 \mathrm{mmHg}, P<0.001)$ and higher CVP $(11.0 \mathrm{cmH} 2 \mathrm{O}$ vs. $8.0 \mathrm{cmH} 2 \mathrm{O}, P<0.001)$, and the multivariable logistic regression analysis showed that CVP, but not MAP, was associated with postoperative AKI within the first 24 hours after cardiovascular surgery (OR: 1.58, 95\% CI: 1.42-1.77, $P<$ 0.001). An increase in the CVP impedes venous return, which leads to the elevated venous pressure and decrease cardiac output. In addition, an increased venous pressure results in an increased renal subcapsular pressure, which reduces blood flow and glomerular filtration rate [Prowle 2014]. This observation was consistent with accumulating evidence suggesting an association between CVP and impaired renal function [Damman 2009; 27]. One study showed that increased CVP, but not MAP, had been associated with impaired and worsening renal function and increased mortality in patients with a broad spectrum of cardiovascular diseases [Damman 2009]. Another study also reported that an increased CVP at the end of cardiac surgery was associated with the development of AKI (OR: 1.42, 95\% CI: 1.35-1.49, $P<0.001$ ), which was especially observed in patients with a CVP $>10 \mathrm{cmH} 2 \mathrm{O}$, and there were more AKI patients need RRT in the high CVP group $(P<0.001)$ [Yang 2018].
The present study has several important limitations. First, this was a single retrospective study lacking a strict protocol for the management of vasoactive and inotropic agents, and the institutions participating in this study might not be representative of the entire ICU community. Furthermore, there were increased heterogeneity in our study, and it would be stronger with homogeneous group, such as CABG only between VIS-max+STS score and postoperative AKI. Nonetheless, this study was the first attempt to evaluate commonly available bedside indices as predictive score for postoperative AKI in the adult population who underwent cardiovascular surgery. Second, the VIS-max was calculated within the first 24 postoperative hours, and patients with AKI on POD-1 could not be predicted by this index. As a solution, to assess the predictive power of VIS-max, we excluded those cases of AKI within 24 postoperative hours from the analysis. In addition, we found that VIS-max+STS score had better discrimination performance than VIS-max, STS score, and EuroSCORE. Third, the VIS-max could only predict cases of postoperative AKI from the second day to the seventh day in our study. However, in addition to being one predictive score, the inclusion of VIS-max to the STS score also could become one tool to guide treatment in patients with postoperative AKI and might assist in determining the start time of RRT. Finally, this study lacked information on long-term prognosis (e.g. one year mortality) and survival analysis in patients with different stages of postoperative AKI. In addition, patients who underwent major vascular surgery did not include abdominal aortic surgery. Consequently, follow-up large prospective studies are needed to confirm this.

In conclusion, this study showed that the inclusion of VIS-max to the risk model of STS score was independently associated with postoperative AKI in adult patients following cardiovascular surgery and complemented existing scores as an early indicator of renal replacement therapy in this population. Therefore, VIS-max, which is easily calculated from routine work, could assist in predicting AKI and need of RRT in high-risk cardiovascular surgery.

\section{REFERENCES}

Badhwar V, et al. 2018. The Society of Thoracic Surgeons Adult Cardiac Surgery Database: 2018 Update on Research: Outcomes Analysis, Quality Improvement, and Patient Safety. Ann Thorac Surg. 106(1): p. 8-13.

Bangalore H, et al. 2017. The Total Inotrope Exposure Score: an extension of the Vasoactive Inotrope Score as a predictor of adverse outcomes after paediatric cardiac surgery. Cardiol Young. 27(6): p. 1146-1152.

Butts RJ, et al. 2012. Comparison of maximum Vasoactive-Inotropic Score and low cardiac output syndrome as markers of early postoperative outcomes after neonatal cardiac surgery. Pediatr Cardiol. 33(4):633-638.

Chang CH, Mehta RH, and Grab JD, et al. 2006. Society of Thoracic Surgeons National Cardiac Surgery Database I. Bedside tool for predicting the risk of postoperative dialysis in patients undergoing cardiac surgery. Circulation. 114: 2208-16.

Che M, et al. 2019. A Clinical Score to Predict Severe Acute Kidney Injury in Chinese Patients after Cardiac Surgery. Nephron. 142(4): p. 291-300. 
Damman K, van Deursen VM, Navis G, et al. 2009. Increased central venous pressure is associated with impaired renal function and mortality in a broad spectrum of patients with cardiovascular disease. J Am Coll Cardiol. 53: 582-8.

DeLong ER, DeLong DM, Clarke-Pearson DL. 1988. Comparing the areas under or more correlated receiver operating characteristic curves: A nonparametric approach. Biometrics. 44:837-45.

Englberger L, Suri RM, Li Z, et al. 2011. Clinical accuracy of RIFLE and acute kidney injury network (AKIN) criteria for acute kidney injury in patients under going cardiac surgery. Crit Care. 15:R16.

Gaies MG, et al. 2014. Vasoactive-inotropic score is associated with outcome after infant cardiac surgery: an analysis from the Pediatric Cardiac Critical Care Consortium and Virtual PICU System Registries. Pediatr Crit Care Med. 15(6): p. 529-37.

Gaies MG, Gurney JG, Yen AH, et al. 2010. Vasoactive-inotropic score as a predictor of morbidity and mortality in infants after cardiopulmonary bypass. Pediatr Crit Care Med. 11:234-238.

Garcia RU, Walters HL, Delius RE, et al. 2016. Vasoactive-inotropic score (VIS) as biomarker of short-term outcomes in adolescents after cardiothoracic surgery. Pediatr Cardiol. 37: 271-7.

Gruenwald CE, McCrindle BW, Crawford-Lean L, et al. 2008. Reconstituted fresh whole blood improves clinical outcomes compared with stored component blood therapy for neonates undergoing cardiopulmonary bypass for cardiac surgery: A randomized controlled trial. J Thorac Cardiovasc Surg. 136:1442-1449.

Haase-Fielitz, A, M. Haase, et al. 2009. Decreased catecholamine degradation associates with shock and kidney injury after cardiac surgery. J Am Soc Nephrol. 20 (6): 1393-403.

Jorge-Monjas P, et al. 2016. Predicting cardiac surgery-associated acute kidney injury: The CRATE score. J Crit Care. 31(1): p. 130-8.

Kellum, JA and Lameire N. 2013. Diagnosis, evaluation, and management of acute kidney injury: a KDIGO summary (Part 1). Crit Care, 2013. 17(1): p. 204.

Kim JW, et al. 2015. Preoperative factors as a predictor for early postoperative outcomes after repair of congenital transposition of the great arteries. Pediatr Cardiol. 36(3):537-542.
Koponen T, et al. 2019. Vasoactive-inotropic score and the prediction of morbidity and mortality after cardiac surgery. Br J Anaesth. 122(4): p. $428-436$.

Lumlertgul N, et al. 2018. Early versus standard initiation of renal replacement therapy in furosemide stress test non-responsive acute kidney injury patients (the FST trial). Crit Care. 22(1): p. 101.

Nadim MK, et al. 2018. Cardiac and Vascular Surgery-Associated Acute Kidney Injury: The 20th International Consensus Conference of the ADQI (Acute Disease Quality Initiative) Group. J Am Heart Assoc. 7(11).

Prowle JR, Kirwan CJ, Bellomo R. 2014. Fluid management for the prevention and attenuation of acute kidney injury. Nat Rev Nephrol. 10:37-47.

Ranucci M, et al. 2018. Validation of renal-risk models for the prediction of non-renal replacement therapy cardiac surgery-associated acute kidney injury. Int J Cardiol. 272: p. 49-53.

Stamm C, Friehs I, Cowan DB, et al. 2002. Dopamine treatment of post ischemic contractile dysfunction rapidly induces calciumdependent proapoptotic signaling. Circulation. 106 (Suppl 1): I290-I298.

Vanmassenhove J, et al. 2017. Management of patients at risk of acute kidney injury. Lancet. 389(10084): p. 2139-2151.

Wernovsky G, Wypij D, Jonas RA, et al. 1995. Postoperative course and hemodynamic profile after the arterial switch operation in neonates and infants. A comparison of low-flow cardiopulmonary bypass and circulatory arrest. Circulation. 92:2226-2235.

Yamazaki Y, et al. 2018. Vasoactive-inotropic score as a predictor of morbidity and mortality in adults after cardiac surgery with cardiopulmonary bypass. J Anesth. 32(2): p. 167-173.

Yang Y, J Ma, et al. 2018. High central venous pressure is associated with acute kidney injury and mortality in patients underwent cardiopulmonary bypass surgery. J Crit Care. 48: 211-215.

Yuan SM, et al. 2019. Acute Kidney Injury after Cardiac Surgery: Risk Factors and Novel Biomarkers. Braz J Cardiovasc Surg. 34(3): p. 352-360. 